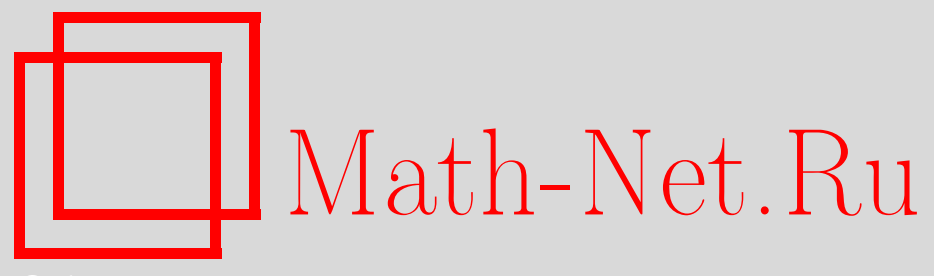

В. П. Маслов, Отрицательная асимптотическая топологическая размерность, новый конденсат и их связь с квантованным законом Ципфа, Матем. заметки, 2006, том 80, выпуск 6, 856-863

DOI: https://doi.org/10.4213/mzm3362

Использование Общероссийского математического портала Math-Net.Ru подразумевает, что вы прочитали и согласны с пользовательским соглашением http://www.mathnet.ru/rus/agreement

Параметры загрузки:

IP : 52.87 .193 .239

26 апреля 2023 г., 17:11:06

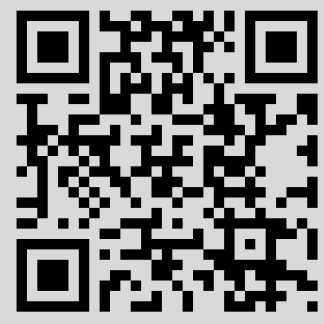




\section{ОТРИЦАТЕЛЬНАЯ АСИМПТОТИЧЕСКАЯ ТОПОЛОГИЧЕСКАЯ РАЗМЕРНОСТЬ, НОВЫЙ КОНДЕНСАТ И ИХ СВЯЗЬ С КВАНТОВАННЫМ ЗАКОНОМ ЦИПФА}

\section{В. П. Маслов}

Вводится понятие веса для асимптотической топологической размерности. Уточняется формула Планка для излучения черного тела. Вводится понятие отрицательной асимптотической топологической размерности (дырочной размерности). Применяется конденсат в дырочной размерности к проквантованному ранее автором закону Ципфа для частотных словарей.

Библиография: 11 названий.

В работе [1] возникло два подхода к ранговым распределениям, аналогичным закону Ципфа.

Первый подход связан с учетом человеческого фактора. Так, зависимость числа проданных товаров от их цены, естественно, отражает восприятие покупателя, и мы не можем обойтись без учета оценки покупателя, которая является виртуальной и неточной величиной. В работах [2], [3] была получена формула

$$
\omega=\operatorname{const}\left(\frac{r}{r_{c}}\right)^{1 / \gamma},
$$

где $\omega$ - цена, $r$ - порядковый номер проданного товара, отсчитываемый от товаров с наиболее низкой ценой, $r_{c}$ - порядковый номер проданного товара, отсчитываемый от товаров с наиболее высокой ценой, так, что $r+r_{c}=N$, где $N$ - число всех проданных товаров. Константа $\gamma$ характеризует рынок товаров длительного пользования, в данном случае американский авторынок: для него $\gamma=5.75855$. В [3] мы приводили график реальных продаж для американского авторынка и теоретической кривой, отвечающей формуле (1).

Отметим, что для товаров длительного пользования цена для покупателя выше, чем выставленная продавцом. Это можно проследить на примере авторынка. Чем дороже автомобиль, тем больше риск, что его угонят, тем больше цена запасных частей в случае поломок и т.п. Следовательно, чтобы избежать этих рисков, нужно покупать страховку и учитывать хлопоты, которые связаны с выплатой страховой суммы, ликвидность, мгновенное падение цены покупки и т.п. Чем дороже автомобиль, тем длиннее "хвост" дополнительных расходов и тем больше риск, который

Работа выполнена при поддержке Российского фонда фундаментальных исследований, грант № 05-01-00824.

(C) В. П. МАСлов, 2006 
определяет его цену для покупателя, учитывающего сопутствующие товары и услуги.

Как уже говорилось в [2], если цена на автомобиль невысокая, особенно если он подержанный, то здесь дополнительный шлейф расходов растет по мере уменьшения цены: увеличивается риск попасть в аварию, дорожный налог на старую машину за рубежом также существенно увеличен, опасность выхода из строя детали возрастает и т.д. Следовательно, цена автомобиля для покупателя с учетом этих обстоятельств складывается из номинальной цены $p_{i}$ плюс цена шлейфа дополнительных расходов.

Аналогично для отдельных акций их частота встречаемости зависит от трейдера, который может выбрать вместо данной акции близкую по типу и цене.

Однако, когда мы рассматриваем ранговое распределение явлений природы таких, как частота выбросов в Чернобыльской АЭС (см. [4]), распределение кратеров на луне, часоту землетрясений, то восприятие их человеком роли играть не может.

Точно так же, когда мы рассматриваем частоту встречаемости "японских свечей" (см. [2]) для большого набора акций или большой частотный словарь, составленный на базе огромного массива текстовых документов, то зависимость ранга от частоты уже не связана с человеческим фактором.

Эти распределения, как мы говорили в [1], связаны с некоторым разрежением спектра, на котором мы остановимся ниже после доказательства теоремы.

Как это обычно рассматривается в математической статистике, относительно значений случайной величины устанавливается упорядоченность по их величине ("порядковая статистика"). Среди чисел $\lambda_{1}, \ldots, \lambda_{s}$ могут быть и равные. В последнем случае их объединяют, складывая при этом отвечающие им "вероятности", т.е. отношение числа "попаданий" на $\lambda_{i}$ к общему числу испытаний. Число равных $\lambda_{i}$, именно $\lambda_{i}=\lambda_{i+1}=\cdots=\lambda_{i+k}$, будем называть кратностъю значения $\lambda_{i}$ и обозначать $q_{i}$ (здесь $q_{i}=k$ ). В нашем рассмотрении к бесконечности стремится как число испытаний $N$, так и $s$.

Пусть $N_{i}$ - число "выпадений" значения $\lambda_{i}: \lambda_{i}<\lambda_{i+1}$; тогда

$$
\sum_{i=1}^{s} \frac{N_{i}}{N} \lambda_{i}=M
$$

где $M$ - математическое ожидание.

Кумулятивная вероятность $\mathscr{P}_{k}$ есть сумма первых $k$ вероятностей в последовательности $\lambda_{i}$ :

$$
\mathscr{P}_{k}=\frac{1}{N} \sum_{i=1}^{k} N_{i}
$$

где $k<s$. Обозначим $N P_{k}=B_{k}$.

Кумулятивная вероятность - это и есть "распределение", а сами значения $N_{i} / N$ - это плотность распределения.

Если все варианты, для которых

$$
\sum_{i=1}^{s} N_{i}=N
$$

и

$$
E-\delta_{N} \leqslant \sum_{i=1}^{s} N_{i} \lambda_{i} \leqslant E, \quad E=M N \leqslant N \bar{\lambda}
$$


где

$$
\bar{\lambda}=\frac{1}{Q} \sum_{i=1}^{s} q_{i} \lambda_{i}, \quad Q=\sum_{i=1}^{s} q_{i}, \quad \delta_{N}=\alpha \bar{\lambda} \ln N,
$$

$\alpha>0$ - константа, равноценны, иначе говоря равновероятны, то в силу теоремы, доказанной автором в [5], [6] (см. также [8]), большинство вариантов будет скапливаться около следующей зависимости "коммулятивной вероятности" $B_{l}=\sum_{i=1}^{l} N_{i}$ :

$$
B_{l}=\sum_{i=1}^{l} \frac{q_{i}}{e^{\beta^{\prime} \lambda_{i}-\nu^{\prime}}-1},
$$

где $\beta^{\prime}$ и $\nu^{\prime}$ определяются из условий

$$
B_{s}=N, \quad \sum_{i=1}^{s} \frac{q_{i} \lambda_{i}}{e^{\beta^{\prime} \lambda_{i}-\nu^{\prime}}-1}=E
$$

если $N \rightarrow \infty$ и $s \rightarrow \infty$.

Оказывается, что в этом случае большинство вариантов будет скапливаться также около следующей зависимости “локальной энергии": $E_{l}=\sum_{i=1}^{l} \lambda_{i} N_{i}$ вблизи

$$
\sum_{i=1}^{l} \frac{q_{i} \lambda_{i}}{e^{\beta^{\prime} \lambda_{i}-\nu^{\prime}}-1}
$$

где $\beta^{\prime}$ и $\nu^{\prime}$ определяются из условий (6), если $N \rightarrow \infty$ и $s \rightarrow \infty$.

Теорема формулируется следующим образом.

Теорема 1. Пусть все варианты наборов $\left\{N_{i}\right\}$ таких, что

$$
E-\delta_{N} \leqslant \sum_{i=1}^{s} N_{i} \lambda_{i} \leqslant E
$$

и выполняется условие (3), равновероятны. Тогда число вариантов $\mathscr{N}$ наборов $\left\{N_{i}\right\}$, удовлетворяющих (3) и (4), для которых выполняется дополнительное соотношение

$$
\frac{1}{\lambda_{l}}\left|\sum_{i=1}^{l} \lambda_{i} N_{i}-\sum_{i=1}^{l} \frac{q_{i} \lambda_{i}}{e^{\beta^{\prime} \lambda_{i}-\nu^{\prime}}-1}\right| \geqslant C_{m}(\alpha) Q^{1 / 2+\varepsilon} \ln N,
$$

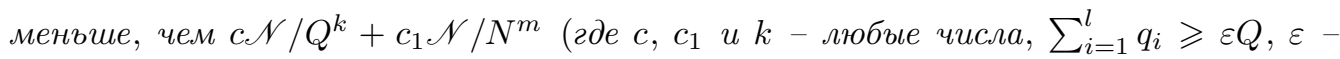
сколь угодно малое, а $C_{m}(\alpha)$ - константа, зависящая только от $m u \alpha$ ).

Обозначения: $\mathscr{M}$ - множество всех наборов $\left\{N_{i}\right\}$, удовлетворяющих условиям (3) и $(4) ; \mathscr{N}\{\mathscr{M}\}$ - число элементов множества $\mathscr{M} ; \mathscr{A}$-подмножество $\mathscr{M}$, удовлетворяющее условию

$$
\frac{1}{\lambda_{l}}\left|E_{l}-\sum_{i=1}^{l} \frac{q_{i} \lambda_{i}}{e^{\beta \lambda_{i}-\nu}-1}\right| \leqslant \Delta,
$$

где $\Delta, \beta, \nu$ - некоторые действительные числа, не зависящие от $l$.

Обозначим

$$
\frac{1}{\lambda_{l}}\left|E_{l}-\sum_{i=1}^{l} \frac{q_{i} \lambda_{i}}{e^{\beta \lambda_{i}-\nu}-1}\right|=S_{l} .
$$


ДоКАЗАТЕЛЬСТво ТЕОРемЫ 1 . Очевидно, что если $\mathscr{M}$ - множество всех наборов $\left\{N_{i}\right\}$, то

$$
\mathscr{N}\{\mathscr{M} \backslash \mathscr{A}\}=\sum_{\left\{N_{i}\right\}}\left(\Theta\left\{E-\sum_{i=1}^{s} N_{i} \lambda_{i}\right\} \delta_{\left(\sum_{i=1}^{s} N_{i}\right), N} \Theta\left\{\left|S_{l}-\Delta\right|\right\}\right) \Theta_{1},
$$

где $\Theta_{1}=\sum_{\left\{N_{i}\right\}} \Theta\left(N_{i} \lambda_{i}-E+\delta_{N}\right), \sum N_{i}=N$. Здесь сумма берется по всем целым $N_{i}, \Theta(\lambda)$ - функция Хевисайда, а $\delta_{k_{1}, k_{2}}$ - символ Кронекера.

Используем интегральные представления

$$
\begin{aligned}
& \delta_{N N^{\prime}}=\frac{e^{-\nu N}}{2 \pi} \int_{-\pi}^{\pi} d \varphi e^{-i N \varphi} e^{\nu N^{\prime}} e^{i N^{\prime} \varphi}, \\
& \Theta(y)=\frac{1}{2 \pi i} \int_{-\infty}^{\infty} d \lambda \frac{1}{\lambda-i} e^{\beta y(1+i \lambda)} .
\end{aligned}
$$

Далее, используя общепринятую регуляризацию, получаем

$$
\int_{0}^{\infty} d M \Theta\left(M-\sum_{i=1}^{s} N_{i} \lambda_{i}\right) e^{-\beta E}=\int_{\sum_{i=1}^{s} N_{i} \lambda_{i}}^{\infty} d E e^{-\beta E}=\frac{1}{\beta} \exp \left\{-\beta \sum_{i=1}^{s} N_{i} \lambda_{i}\right\} .
$$

Обозначим

$$
Z(\beta, N)=\sum_{\left\{N_{i}\right\}} \exp \left\{-\beta \sum_{i=1}^{s} N_{i} \lambda_{i}\right\}
$$

где сумма берется по всем $N_{i}$ таким, что $\sum_{i=1}^{s} N_{i}=N$,

$$
\zeta_{l}(\nu, \beta)=\prod_{i=1}^{l} \xi_{i}(\nu, \beta), \quad \xi_{i}(\nu, \beta)=\frac{1}{\left(1-e^{\nu-\beta \lambda_{i}}\right)^{q_{i}}}, \quad i=1, \ldots, l .
$$

В силу (10)

$$
Z(\beta, N)=\frac{e^{-\nu N}}{2 \pi} \int_{-\pi}^{\pi} d \alpha e^{-i N \alpha} \zeta_{s}(\beta, \nu+i \alpha)
$$

отсюда

$$
\begin{aligned}
\mathscr{N}\{\mathscr{M} \backslash \mathscr{A}\} \leqslant \mid & \frac{e^{-\nu N+\beta E}}{i(2 \pi)^{2}} \int_{-\pi}^{\pi}\left[e ^ { - i N \varphi } \sum _ { \{ N _ { j } \} } \operatorname { e x p } \left\{\left(-\beta \sum_{j=1}^{s} N_{j} \lambda_{j}\right)\right.\right. \\
& \left.\left.+(i \varphi+\nu) N_{j}\right\}\right] d \varphi \mid \cdot \Theta\left(\left|S_{l}-\Delta\right|\right) \Theta_{1}, \quad \sum N_{i}=N,
\end{aligned}
$$

где $\beta$ и $\nu$ - такие действительные параметры, для которых ряд сходится.

Оценивая правую часть, пронеся модуль под интеграл и затем под знак суммы и интегрируя по $\varphi$, получим

$$
\mathscr{N}\{\mathscr{M} \backslash \mathscr{A}\} \leqslant \frac{e^{-\nu N+\beta E}}{2 \pi} \sum_{\left\{N_{i}\right\}} \exp \left\{-\beta \sum_{i=1}^{s} N_{i} \lambda_{i}+\nu N_{i}\right\} \cdot \Theta\left(\left|S_{l}-\Delta\right|\right) \Theta_{1} .
$$

Из неравенства для гиперболического косинуса $\operatorname{ch}(x)=\left(e^{x}+e^{-x}\right) / 2$ :

$$
\operatorname{ch}\left(x_{l}\right) \geqslant e^{\delta} \quad \forall x_{l}: \quad\left|x_{l}\right| \geqslant \delta \geqslant 0,
$$


следует, что для всех положительных $c$ и $\Delta$ выполняется неравенство (ср. [5])

$$
\Theta\left(\left|S_{l}-\Delta\right|\right) \leqslant e^{-c \Delta} \operatorname{ch}\left(c \sum_{i=1}^{l} \lambda_{i} N_{i}-c \psi_{\nu \beta}\right)
$$

где

$$
\psi_{\nu \beta}=\sum_{i=1}^{l} \frac{q_{i} \lambda_{i}}{e^{\beta \lambda_{i}-\nu}-1}
$$

Получаем

$$
\begin{aligned}
\mathscr{N}\{\mathscr{M} \backslash \mathscr{A}\} \leqslant & e^{-c \Delta} \exp (\beta E-\nu N) \Theta_{1} \\
& \times \sum_{\left\{N_{i}\right\}} \exp \left\{-\beta \sum_{i=1}^{s} N_{i} \lambda_{i}+\nu N_{i}\right\} \operatorname{ch}\left(\sum_{i=1}^{l} c \lambda_{i} N_{i}-c \psi_{\nu \beta}\right) \\
= & e^{\beta E} e^{-c \Delta}\left(\zeta_{l}(\nu, \beta-c) \exp \left(-c \psi_{\nu \beta}\right)+\zeta_{l}(\nu, \beta+c) \exp \left(c \psi_{\nu \beta}\right)\right) \Theta_{1} .
\end{aligned}
$$

Применим к $\zeta_{l}(\nu, \beta \pm c)$ формулу Тейлора. Существует $\gamma<1$ такое, что

$$
\ln \left(\zeta_{l}(\nu, \beta \pm c)\right)=\ln \zeta_{l}(\nu, \beta) \pm c\left(\ln \zeta_{l}\right)_{\beta}^{\prime}(\nu, \beta)+\frac{c^{2}}{2}\left(\ln \zeta_{l}\right)_{\beta}^{\prime \prime}(\nu, \beta \pm \gamma c)
$$

Очевидно, что

$$
\frac{\partial}{\partial \beta} \ln \zeta_{l} \equiv-\psi_{-\nu, \beta}
$$

Положим $c=\Delta / D(\nu, \beta)$, где $D(\nu, \beta)=\left(\ln \zeta_{l}\right)_{\beta}^{\prime \prime}(\nu, \beta)$ больше нуля при всех $\beta$, причем монотонно убывает по $\beta$. Правая часть соотношения (18) не будет превосходить

$$
e^{-\nu N+\beta E} \zeta_{l}(\nu, \beta) \exp \left\{-\frac{\Delta_{l}^{2}}{|D(\nu, \beta)|}+\frac{\Delta_{l}^{2} D(\nu, \beta-\gamma \Delta / D(\nu, \beta))}{2(D(\nu, \beta))^{2}}\right\} \Theta_{1} .
$$

Накладывая на $\Delta$ ограничения вида

$$
D\left(\nu, \beta-\frac{\Delta}{D(\nu, \beta)}\right) \leqslant(2-\varepsilon) D(\nu, \beta)
$$

где $\varepsilon>0$, и имея в виду, что $D(\nu, \beta)$ монотонно убывает по $\beta$, получим

$$
\mathscr{N}(\mathscr{M} \backslash \mathscr{A}) \leqslant e^{\beta E} e^{-\nu N} \zeta_{l}(\nu, \beta) e^{-\varepsilon \Delta^{2} / D(\nu, \beta)} .
$$

В силу того, что $e^{\nu-\beta \lambda_{i}}<1$, для всех $\alpha \neq 2 \pi n$, где $n-$ целое число, выполняются строгие неравенства

$$
\left|\xi_{i}(\nu+i \alpha, \beta)\right|<\xi_{i}(\nu, \beta), \quad\left|\zeta_{s}(\nu+i \alpha, \beta)\right|<\zeta_{s}(\nu, \beta)
$$

Поэтому в интервале от $-\pi$ до $\pi$, по которому берется интеграл (13), вклад дает только окрестность точки $\alpha=0$. Вычисляя интеграл (13) методом Лапласа с точностью до $N^{-m}$ и применяя оценки для $D$ и $D_{1}$ из [5], получим утверждение теоремы. 
ЗАмЕЧАНИЕ. Если выполняется условие

$$
E \geqslant \frac{N}{Q} \sum_{i=1}^{s} q_{i} \lambda_{i}
$$

то $\sum_{i=1}^{s} \lambda_{i} N_{i} \geqslant E$ и $\sum_{i=1}^{s} \lambda_{i} N_{i} \leqslant E+\delta_{N}$. При этом получается $\beta<0$.

Теперь перейдем к асимптотической топологической размерности.

Теория асимптотической топологической размерности дискретных объектов, которые мы рассмотрим ниже в простейшем варианте, бурно развивается после известной работы М. Громова [10] (см. также [11]).

Рассмотрим прямую, плоскость и трехмерное пространство. На прямой мы отложим точки $i=0,1,2, \ldots$, на осях координат $x, y$ плоскости точки $x=i=0,1,2, \ldots$, $y=j=0,1,2, \ldots$. Мы сопоставим этому множеству точек $(i, j)$ точки на прямой натуральный ряд $l=1,2, \ldots$ с точностью до квантовой постоянной решетки $\chi$.

Согласно определению Громова асимптотическая (топологическая) размерность этой решетки равна 2.

Сопоставим каждой точке пару точек $i$ и $j$ по правилу $i+j=l$. Число таких точек $n_{l}$ равно $l+1$. Если еще положить на оси $z=k=0,1,2, \ldots$, т.е. положим $i+j+k=l$. В этом случае число точек $q_{l}$ будет равно $q_{l}=(l+1)(l+2) / 2$.

Если положить в формуле (8) $\lambda_{i}=l$, то в трехмерном случае каждому $i$ будет соответствовать $(l+1)(l+2) / 2$ равных между собой $\lambda_{l}=l$ (это кратности или "вырожденности" спектра осциллятора). Формула (8) в этом частном случае будет выглядеть следующим образом:

$$
\begin{aligned}
N_{l} & =\operatorname{const} \sum_{i=0}^{l} \frac{(i+1)(i+2)}{2\left(e^{\beta i-\nu}-1\right)}, \\
\Delta N_{i} & =\operatorname{const} \frac{(i+1)(i+2)}{2\left(e^{\beta i-\nu}-1\right)} \Delta i, \quad \Delta i=1, \\
\Delta E_{i} & =\operatorname{const} \frac{i(i+1)(i+2)}{2\left(e^{\beta i-\nu}-1\right)} \Delta i \quad \text { при больших } i, \quad \frac{\Delta i}{i} \rightarrow 0, \\
d E & =\text { const } \frac{\omega^{3} d \omega}{e^{\beta \omega}-1}, \quad \beta=\frac{h}{T}
\end{aligned}
$$

(ср. [12; формула (60.4)]).

Этим самым, мы несколько уточняем знаменитую формулу Планка для излучения черного тела.

Нетрудно проверить для $D$-мерного случая, что последовательность весов (кратностей) числа вариантов $i=\sum_{k=1}^{D} m_{k}$, где $m_{k}$ - любые натуральные числа, имеет вид факториальной степени $D$

$$
q_{i}(D)=\mathrm{const} \frac{(i+D-1) !}{i ! D !},
$$

константа зависит от $D$.

Таким образом, формула (8) для любого $D$ имеет вид

$$
N_{l}=\mathrm{const} \sum_{i=1}^{l} \frac{q_{i}(D)}{e^{\beta i}-1} .
$$


Для натурального ряда мы имеем последовательность весов $q_{i}$ (или просто вес) вида (25).

Нетрудно продолжить и наш весовой ряд на произвольный случай, заменив факториалы на Г-функции, при этом положим $D$ отрицательным.

Это отрицательная топологическая размерность квантованного пространства (решетки).

Отметим, что положительную размерность $3 N$ ( $N$ - число частиц) часто связывают с числом частиц. Отрицательную асимптотическую топологическую размерность будем называть дърочной. Отрицательные факториальные степени рассматривал еще Стирлинг.

Если $D>1$, то в спектре осциллятора происходит сгущение при $i \rightarrow \infty$ достаточно малого возмущения и кратности расщепляются, т.е. с возрастанием $i$ спектр становится все гуще. Отрицательное $D$ означает при $i \rightarrow \infty$ имеет место сильное разрежение спектра (константа в формуле (26) должна быть достаточно большой).

При отрицательном целом $D$ члены $i=1,2,3, \ldots,-D$ обращаются в бесконечность. Это значит, что на эксперименте они очень велики. Это сразу позволяет определить отрицательную размерность решетки, отвечающую данной задаче. Имеет место новый конденсат, который возможен при малых $\beta$.

Закон Ципфа сформулирован для логарифма ранга (номера) слов в частотных словарях. Поскольку мы приводим формулу непосредственно для самих рангов, а не для их логарифмов (аналог - классическое действие и волна де Бройля), то называем эту процедуру квантованием закона Ципфа (тем более, что обратное действие называется деквантованием [13]).

Частотный словарь составляется из текстов определенного массива. Каждому слову словаря сопоставлено число его встречаемости в исходном массиве текстов. Будем обозначать это число $\omega_{i}$, а число слов, отвечающих этому числу встречаемости, $n_{i}$. Сумма всех $n_{i}$ равна объему всего словаря, а сумма произведений $\omega_{i} n_{i}-$ объему всего массива текстов, на основе которого составлен словарь.

Слова, которые встречаются в массиве текстов один раз, составляют примерно $1 / 3$ всего частотного словаря, а это - конденсат. В силу того, что было сказано выше, для словаря $D \sim-1$. Значит, при $\beta \ll 1, \nu \sim 1$

$$
N_{l}=\mathrm{const} \sum_{i=2}^{l} \frac{1}{i(i-1)\left(e^{\beta i-\nu}-1\right)} \sim \operatorname{const} \int^{\omega} \frac{d \omega}{\omega(\omega-1)\left(e^{\beta \omega-\nu}-1\right)}
$$

для $\omega=l$.

Параметр $\beta$ должен быть порядка $1 / \omega_{\max }, \omega_{\max }$ - частота наиболее часто встречающегося слова, если отброшены так называемые "стоп-слова" - служебные слова типа $u, a, н о$ и т.п. Пусть объем словаря без стоп-слов и без конденсата $(\omega>1)$ равен $N$, тогда ранг (номер) слова равен $r=N-N_{l}$. Для средних и малых частот формула имеет вид

$$
r=c_{1} \ln \left(1+\frac{1}{\omega}\right)+c_{2}
$$

$c_{1}, c_{2}$ - константы.

При $\beta \rightarrow 0, \omega \rightarrow \infty, \beta \omega \rightarrow$ const формула (27) переходит в логарифмических координатах в соотношение Ципфа вида

$$
\ln \left(N-N_{l}\right) \sim-\ln \omega .
$$


В этом смысле формула (27) является ее "квантовым" продолжением.

Для "японских свечей" (см. [2]) размерность равна 1 при распределении для 30 акций.

\section{СПИСОК ЦИТИРОВАННОЙ ЛИТЕРАТУРЫ}

[1] V.P. Maslov, "Quantum linguistic statistics", Russ. J. Math. Phys., 13:3 (2006), 315-325.

[2] В. П. Маслов, Т. В. Маслова, "О законе Ципфа и ранговых распределениях в лингвистике и семиотике", Матем. заметки, 80:5 (2006), 718-732.

[3] В. П. Маслов, "О минимизации статистического риска покупок на рынке недвижимости и товаров длительного пользования", Докл. РАН, 411:6 (2006).

[4] В. П. Маслов, "Фазовые переходы нулевого рода и квантование закона Ципфа", ТМФ, 150:1 (2007), 121-141.

[5] В. П. Маслов, "Нелинейное среднее в экономике", Матем. заметки, 78:3 (2005), 377395.

[6] В.П. Маслов, “Об одной общей теореме теории множеств, приводящей к распределению Гиббса, Бозе-Эйнштейна, Парето и закону Ципфа-Мандельброта для фондового рынка", Матем. заметки, 78:6 (2005), 870-877.

[7] В. П. Маслов, "Уточнение закона Ципфа для частотных словарей", Докл. PAH, 405:5 (2005), 591-594.

[8] В. П. Маслов, "Закон "отсутствия предпочтения" и соответствующее распределение в частотной теории вероятностей", Матем. заметки, 80:2 (2006), 220-230.

[9] В.П. Маслов, "Закон больших уклонений в теории чисел. Вычислимая функция от многих аргументов и декодирование", Докл. РАН, 404:6 (2005), 731-736.

[10] M. Gromov, "Asymptotic invariants of infinite groups", Geometric Group Theory, Vol. 2 (Sussex, 1991), London Math. Soc. Lecture Note Ser., 182, Cambridge Univ. Press, Cambridge, 1993, 1-295.

[11] A. Dranishnikov, J. Smith, "Asymptotic dimension of discrete groups", Fund. Math., 189:1 (2006), 27-34; arXiv: math.GT/0603055v1.

[12] Л. Д. Ландау, Е. М. Лившиц, Теоретическая физика. Том 5. Статистическая физика, Наука, М., 1976.

[13] O. Viro, "Dequantization of real algebraic geometry on logarithmic paper", 3rd European Congress of Mathematics, Vol. I (Barcelona, 2000), Progr. Math., 201, Birkhäuser, Basel, 2001, 135-146. 